\title{
Estudo radiográfico contrastado do tempo de trânsito gastrintestinal em tucanos toco (Ramphastos toco)
}

\section{Evaluation of gastrointestinal tract transit times using barium sulfate suspension in toco toucan (Ramphastos toco)}

\author{
Juliana Macedo Magnino Silva, ${ }^{*}$ André Luiz Quagliatto Santos, ${ }^{*}$ Líria Queiroz Luz Hirano, ${ }^{*}$ Heloísa Castro Pereira, ${ }^{*}$ \\ Arthur Paulino Sanzo Kaminishi*
}

\begin{abstract}
Resumo
O exame radiográfico tem-se tornado um procedimento de rotina no diagnóstico de aves na clínica veterinária. Em aves de cativeiro, osulfato de bário é utilizado como contraste para observar a conformação das vísceras que compõem o trato digestório e definir o tempo de passagemdo alimento. Objetivou-se determinar o tempo de trânsito gastrintestinal(TTGI) em cincotucanos toco (Ramphastos toco) utilizando radiografias contrastadas com suspensão de sulfato de bário a $100 \%$ na dose de $5 \mathrm{ml} / \mathrm{kg}$. As aves foram radiografadas na posição ventrodorsale as tomadas radiográficas foram feitas nos tempos zero, cinco, 15, 30, 40, 60, 70, 90, $135,225,255,310,315,345$ e 355 minutos.A técnica utilizada permitiu a visibilização da passagem do contraste pelas vísceras e determinou o TTGI em tucanos toco. O tempo médio de permanência do sulfato de bário nesta espécie foi de $334 \pm 20$ minutos.
\end{abstract}

Palavras-chave: Ramphastos toco, trato gastrintestinal, radiografia, sulfato de bário.

\begin{abstract}
Radiography has become a routine diagnostic procedure of avian patients in veterinary medicine. In pet and captive avian species, barium sulfate suspension has been used to assessgastrointestinalviscera, as well to observe gastrointestinal transit. The purpose of this study was to determine gastrointestinal transit (GIT) times in toco toucan using $5 \mathrm{mg} / \mathrm{kg}$ of a $100 \%$ barium sulfate suspension. Whole-body ventrodorsal view radiographs were taken at zero, five, 15, 30, 40, 60, 70, 90, 135, 225, 255, 310, 315, 345 and 355 minutes. The technique used in this study permitted the visibilization of barium sulfate suspension through gastrointestinal viscera and allowed the determination ofGIT.The mean permanence time of barium sulfate in toco toucan was $334 \pm 20.12$ minutes.
\end{abstract}

Keywords: Ramphastos toco, gastrointestinal tract, radiography, barium sulfate.

\section{Introdução}

O exame radiográfico tem-se tornado um procedimento de rotina no diagnóstico de aves na clínica veterinária. O menor porte desses animais permite a visibilizaçãode órgãos e vísceras em uma única radiografia e os sacos aéreos promovem um contraste negativo na imagem, delineando os outros órgãos celomáticos (Vink-Noteboomet al., 2003).

Radiografias contrastadas com sulfato de bário são a melhor alternativa para fornecer informações sobre a morfologia, obstrução e enfermidades do trato gastrintestinal (Chen, 1999). Em aves de cativeiro, este contraste tem sido utilizado para observar estruturas e determinar o tempo de trânsito alimentar (Blochet al., 2010).

Relatos sobre o tempo de trânsito gastrintestinal (TTGI) utilizando radiografias contrastadas com sulfato de bário em peixes (Barbieriet al., 1998), mamíferos (Campbell et al., 2004), répteis (Santos et al., 2010) e aves (Mcmillan, 1994), servem de referência para comparar as variações anatômicas e fisiológicas do trato digestório das diferentes espécies animais.
De acordo com Hoferet al. (1982), estudos sobre tempo e motilidade do sistema digestório contribuempara determinar a taxa de consumo alimentar de populaçõesanimais. Magnuson (1969)relacionou o estímulo da fome e o apetite com a quantidade de alimento noestômago e Elliot (1975) estabeleceu a relação entre a capacidade máxima de enchimento gástrico e o tempo de esvaziamento em diferentes temperaturas.

O conhecimento da passagem alimentarpelo trato digestório é importante para definir o manejo nutricional em cativeiro e formular dietas condizentes com a fisiologia digestivade cada espécie. Assim, objetivou-se determinar o tempo de trânsito gastrintestinal em tucanos toco (Ramphastos toco) utilizando radiografia contrastada com suspensãode sulfato de bário a $100 \%$.

\section{Material e métodos}

O experimento foi conduzido no Laboratório de Ensino e Pesquisa em Animais Silvestres (LAPAS) e Hospital Veterinário da Universidade Federal de Uberlândia, após parecer favorável

\footnotetext{
* Laboratório de Ensino e Pesquisa em Animais Silvestres - Universidade Federal de Uberlândia. Rua Piauí, s/n, Bloco 4S, Bairro Jardim Umuarama. Uberlândia, MG. CEP 38400-902. Email: juliana.magnino@gmail.com

*Autor para correspondência: Juliana Macedo Magnino Silva.
} 
da comissão de ética na utilização de animais da Universidade Federal de Uberlândia, protocolo CEUA/UFU 103/10.

Os tucanos toco passaram por um exame físico de acordo com Werther (2008), foram alojados em viveiros telados, com acesso a luz solar e alimentados com frutas picadas, ração comercial e água ad libitum. Foram utilizados cinco espécimes adultos, hígidos, de ambos os sexos,provenientes da apreensão do tráfico de animais silvestres em dezembro de 2009.

As aves foram alimentadas quatro horas antes da administração do contraste de acordo com Mcmillan (1994). Utilizou-se o sulfato de bário a $100 \%$ (Bariogel $\circledast$ ) em suspensão, na dose de $5 \mathrm{ml} / \mathrm{kg}$ (Delafiori et al., 2009), administrado via oral por meio de uma sonda esofágica descartável diretamente no proventrículo.

Após administração do sulfato de bário, as aves foram contidas manualmente com o auxílio de luvas raspa de couro e radiografadas na posição ventrodorsal, conforme Bloch et al. (2010) no aparelho de raio X Triplunix $® 800 \mathrm{~mA}$ regulado para $52 \mathrm{kv}, 50 \mathrm{~mA}$ e tempo de exposição de 0,032 segundo,sem utilização da mesa bucky. As tomadas radiográficas foram feitas nos tempos, em minutos, zero, cinco, 15, 30, 40, 60, 70, 90, $135,225,255,310,315,345$ e 355.

Foi realizada a estatística descritiva, por meio dos testes de média e desvio-padrão dos tempos de preenchimento e esvaziamento das vísceras gastrintestinais pelo contraste (Silvestre, 2001).

A terminologia adotada neste trabalho está de acordo com a Nomina Anatomica Avium (BAUMEL et al., 1993).

\section{Resultados e discussão}

Neste estudo, realizaram-se tomadas radiográficas com vista ventrodorsal, o que possibilitou a visibilização do proventrículo, ventrículo, duodeno, jejuno, íleo e cólon-reto dos tucanos toco. A utilização desse posicionamento foi embasada no estudo de Bloch et al.(2010), que radiografaram pombos domésticos (Columbalivia) nesta mesma posição e obtiveram imagens satisfatórias em detrimento dos resultados relatados por Vink-Nooteboom et al. (2003), que utilizaram a posição laterolateral para radiografar papagaios verdadeiro (Amazonas aestiva) e conseguiram visibilizar apenas o proventrículo e o ventrículo. Além de dificultar a identificação da alça duodenal, a nitidez das imagens radiográficas obtidas a partir da posiçãolaterolateral é prejudicada pela localização dossacos aéreos e pelasuperposição das asas e dos membros pelvinossobre a cavidade celomática.

Sabe-se que a manipulação prolongada da ave para produção das tomadas radiográficas induz ao estresse e pode causar redução na taxa de passagem (Vink-Nooteboomet al., 2003). Portanto utilizou-se a contenção manual das aves, que demonstrou ser um método prático e seguro quando comparado aos agentes anestésicos, que alteram parâmetros fisiológicos do organismo como a frequência cardíaca e respiratória, além de reduzir a motilidade gastrintestinal (Krautwaldet al., 1992; McMillan, 1994). Já o uso de poleiros envoltos por caixas de acrílico não impede a movimentação do animal durante a tomada radiográfica, comprometendo a qualidade das imagens e aumentando a necessidade de repetições das radiografias, que também poderiam ser um fator causador de estresse (Vink-Nooteboom et al., 2003).

O preenchimento do contraste no proventrículo e ventrículo foi imediato em Ramphastos toco, e procedeu-se da mesma maneira em avestruzes (Struthiocamelus), (Wencke e Kirberger, 2003) pombos domésticos (Bloch, et al., 2010), tucanos-de-bico-verde (Ramphastosdicolorus) (Delafiori et al., 2009) e papagaios verdadeiro (Vink-Nooteboomet al.,2003). O esvaziamento do proventrículo foi imediato em tucanos toco, similar ao reportado em avestruzes (Wencke e Kirberger, 2003)e pombos domésticos (Bloch, et al., 2010),enquanto em tucanos-de-bico-verde (Delafiori et al.,

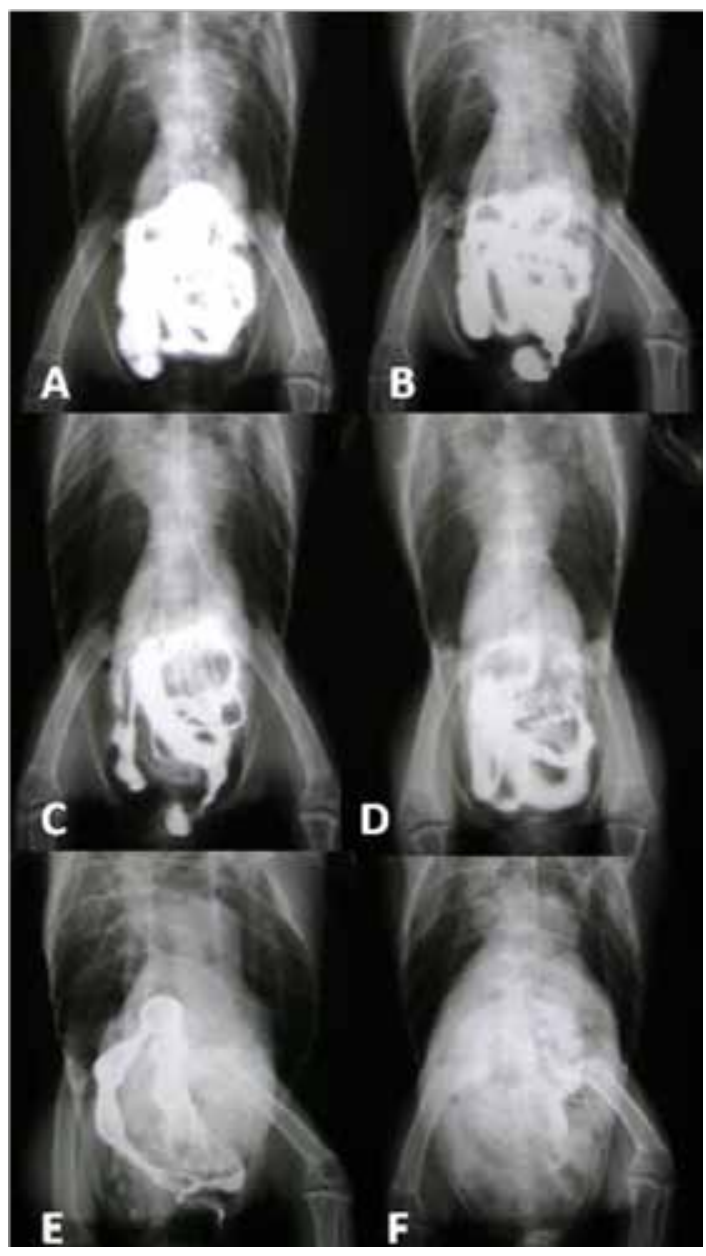

Figura 1: Imagens radiográficas em projeção ventrodorsal da passagem do sulfato de bário nos segmentos do trato gastrintestinal em Ramphastos toco. A: Preenchimento do duodeno, jejuno e íleo cinco minutos após aplicação do contraste. B: Preenchimento do cólon-reto 15 minutos após administração do contraste. C: Duodeno, jejuno, íleo e cólonreto preenchidos com sulfato de bário aos 90 minutos. D: Duodeno, jejuno, íleo preenchidos com sulfato de bário aos 255 minutos. E: Esvaziamento do duodeno 285 minutos após aplicação do contraste. F: Eliminação total do sulfato de bário no trato gastrintestinal após 345 minutos.

2009) e papagaios verdadeiro (Vink-Nooteboomet al., 2003), a passagem do contraste demorou em média, 37,5 e 60 minutos, respectivamente.

No ventrículo, o esvaziamento do contraste demorou cerca de 300 minutos em avestruzes (Wencke e Kirberger, 2003), 420 em pombos (Blochet al., 2010), 61,66 em tucanos-de-bico-verde (Delafiori et al., 2009), 180 em papagaios (Vink-Nooteboomet al., 2003), cinco em canários (Serinus canarius) e 45 em faisões (Phasianus colchicus) (Mcmillan, 1994). Esses dados diferem ao observado em tucanos toco, nos quais o esvaziamento foi imediato em quatro das cinco aves estudadas, sendo que o animal 5 teve o sulfato de bário retido no ventrículo durante 15 minutos. O tempo de passagem do contraste pelas demais estruturas do tubo digestório está indicado na Tabela 1. 
Tabela 1: Tempo de preenchimento, esvaziamento, média e desvio-padrão (D.Pad), em minutos, da passagem da suspensão de sulfato de bário a $100 \%$ pelo trato digestório de cinco exemplares de Ramphastos toco. Uberlândia, 2010

\begin{tabular}{|c|c|c|c|c|c|c|c|c|c|c|}
\hline \multirow{2}{*}{ Animal } & \multicolumn{5}{|c|}{ PREENCHIMENTO } & \multicolumn{5}{|c|}{ ESVAZIAMENTO } \\
\hline & Provent. & Ventrículo & Duodeno & Jejuno/ìleo & Cólon-reto & Provent. & Ventrículo & Duodeno & Jejuno/íleo & Cólon-reto \\
\hline 1 & - & - & 5 & 15 & 135 & - & - & 195 & 325 & 355 \\
\hline 2 & - & - & 5 & 5 & 5 & - & - & 45 & 225 & 310 \\
\hline 3 & - & - & 5 & 5 & 15 & - & - & 285 & 345 & 345 \\
\hline 4 & - & - & 5 & 5 & 15 & - & - & 60 & 285 & 315 \\
\hline 5 & - & - & 5 & 5 & 15 & - & 15 & 285 & 345 & 345 \\
\hline Média & 0 & 0 & 5 & 7 & 37 & 0 & 3 & 174 & 305 & 334 \\
\hline D. Pad. & 0 & 0 & 0 & 4,47 & 54,95 & 0 & 6,71 & 116,96 & 50,99 & 20,12 \\
\hline
\end{tabular}

* O tempo de preenchimento ou esvaziamento do contraste foi imediato à aplicação.

Algumas variações foram observadas entre os exemplares em relação ao preenchimento e esvaziamento das diferentes vísceras do trato gastrintestinal. Esse fato pode ser explicado por Heuser (1945), que mencionou que uma variação individual relacionada com a anatomia, metabolismo e temperamento do animal pode desencadear diferenças no tempo de passagem do alimento pelo sistema gastrintestinal, sendo essa a provável causa das diferenças de tempo de migração do contraste observadas entre as aves, como por exemplo, a retenção mais prolongada do sulfato de bário no ventrículo do animal 5 , uma vez que todos os outros fatores foram constantes e a mesma metodologia foi utilizada para todas as aves.

Além disso, as variações na passagem do contraste pelo tubo digestório podem ser explicadas pelas diferenças na anatomia do trato gastrintestinal entre as espécies de aves, como ausência de papo em tucanos, canários (Ritchie et al., 1994) e avestruzes (Cooper e Mahroze, 2004) e ausência de ceco em tucanos (Cornelissen e Ritchie, 1994). A taxa de passagem do alimento pode ser influenciada pelo tamanho dos intestinos (Lilijahet al.,1985) e pela idade, tamanho e tipo de dieta (Mcmillan, 1994). Tempos de trânsito curtos ocorrem em aves menores, com dietas

\section{Referências}

BARBIERI, R. L.; LEITE, R. G.; HERNANDEZ-BLAZQUEZ, F.J. Morphologie du tract digestif du curimbatá, Prochilodus scrofa. I. Anatomie Intestinale. Ciência e Cultura, v. 41, n. 1, p. 61-70, 1998. BAUMEL,J. J.; KING, A. S.; BREAZILE, J. E.; EVANS, H. E.; VANDEN BERGE, J. C. Handbook of avian anatomy: Nomina Anatomica Avium. 2. ed. Cambridge: Nuttall Ornithological Club 23, 1993. 779 p.

BLOCH, R. A.; CRONIN, K.; HOOVER, J. P.; PECHMAN, R. D.; PAYTON, M. E. Evaluation of gastrointestinal tract transit times using barium-impregnated polyethylene spheres and barium sulfate suspension in a domestic pigeon (Columbia livia) model. Journal of Avian Medicine and Surgery, v. 24, n. 1, p. 1-8, 2010.

CAMPBELL, J. L.; WILLIAMS, C. V.; EISEMANN, J.H. Characterizing Gastrointestinal Transit Time in Four Lemur Species Using Barium-Impregnated Polyethylene Spheres (BIPS). American Journal of Primatology, v. 64, p. 309-321, 2004. pouco consistentes, enquanto a passagem é lenta em aves obesas, granívoras e em filhotes (Mcmillan, 1994).

O tempo médio de TTGI em tucanos toco foi de $334 \pm 20$ minutos. Resultados semelhantes foram relatados por VinkNooteboom et al. (2003) em papagaios (300 minutos) e Mcmillan (1994) em falcões-de-cauda-vermelha (Buteo jamaicensis)(360 minutos). Em avestruzes e pombos, a permanência do contraste no trato gastrintestinal foi maior, com uma média de 960 (Wencke e Kirberger, 2003) e 1440 (Bloch et al., 2010) minutos, respectivamente. Entretanto, outros trabalhos apresentaram tempos médios menores que os observados em Ramphastos toco, como no caso do realizado com periquitos australianos (Melopsittacus undulatus), faisões e tucanos-de-bico-verde, que relataram TTGI de até 240 minutos (Delafiori et al., 2009).

\section{Conclusão}

A técnica de radiografia contrastada com suspensão de sulfato de bário a $100 \%$ permitiu a visibilidade das vísceras do trato digestório e a determinação do tempo de trânsito do trato gastrintestinal em Ramphastos toco. O tempo médio de TTGI em tucanos toco foi de $334 \pm 20$ minutos.

CHEN, J. Reassessment of barium radiographic examination in diagnosing gastrointestinal diseases. World Journal of Gastroenterology, v. 5, n. 5, p. 383-387, 1999.

COOPER, R. G.; MAHROZE, K. M. Anatomy and physiology of the gastro-intestinal tract and growth curves of the ostrich (Struthio camelus). Animal Science Journal, v. 75, p. 491-498, 2004.

CORNELISSEN, H.; RITCHIE, B. W. Ramphastidae In: RITCHIE B. W, HARRISON G. J, HARRISON L. (Ed.). Avian Medicine, Principles and Application. Lake Worth, Florida: Wingers Publishing Inc., 1994, p.127.

DELAFIORI, D. M.; CAVALCANTE, M. K.; GUEDES, P. M.; DELAFIORI, R. E.; NETO, F. D. Q.; TAKAESU, A. Y.; GOMES, M. S. Estudo radiográfico contrastado do sistema digestório de tucanos-de-bico-verde (Ramphastos dicolorus). Nosso Clínico, v. 12, n. 69, p. 38-42, 2009.

ELLIOTT, J. M. Number of meals in a day, maximum weight of food consumed in a day and maximum rate of feeding for brown trout Salmo trutta L. Freshwater Biology, v. 5, n. 2, p. 294-303, 1975. 
HEUSER, G. F. The rate of passage of feed from the crop of the hen. Poultry Science, v. 24, n. 1, p. 20-24, 1945.

HOFER, R.; FORSTNER, H.; RETTENWANDER, R. Duration of gut passage and its dependence on temperature and food consumption in roach Rutilus rutilus L.: Laboratory and field experiments. Journal of Fish Biology, v. 20, n. 3, p. 289-299, 1982.

KRAUTWALD,M. E.; TELLHELM, B.; HUMMEL, G. (Ed.). Atlas of Radiographic Anatomy and Diagnosis of Cage Birds. Verlag Paul Parey, Hamburg, Germany, 1992, p. 31-35.

LILIJAH, C.; SPERBER, I.; MARKS, H. L. Postnatal growth and organ development in Japanese quail selected for high growth rate. Growth, Hulls Cove, v. 49, n. 2, p. 51-62, 1985.

MAGNUSON, J. J. Digestion and food consumption by skipjack tuna Katsuwonus pelamis. Transactions of the American Fishery Society, v. 98, n. 1, p. 379-392, 1969.

MCMILLAN, M. C. Imaging Techniques. In: RITCHIE B. W, HARRISON G. J, HARRISON L. (Ed.). Avian Medicine, Principles and Application. Lake Worth, Florida: Wingers Publishing Inc., 1994, p. 256-259.
RITCHIE, B. W.; HARRISON, G. J.; HARRISON, L. R. Avian Medicine: Principles and application,Wingers Publishing, Lake Worth, 1994.

SANTOS, A. L. Q.; FERREIRA, C. G.; PINTO, J. G. S.; LIMA, C. A P.; VIEIRA, L. G. BRITO, F. M. M. Radiographic anatomy aspects and gastrointestinal transit time in Podocnemisunifilistroschel, 1848 (Testudines, Podocnemididae). Acta Scientiarum. Biological Sciences, v. 32, n. 4, p. 431-435, 2010.

SILVESTRE, A. L. Análise de dados e estatística descritiva. São Paulo: Editora Escolar, 2001.

VINK-NOTEBOOM, M.; LUMEIJ, J. T.; WOLVVEKAMP, W. T. C. Radiography and image-intensified fluoroscopy of barium passage through the gastrointestinal tract in six healthy amazon parrots (Amazona aestiva). Veterinary Radiology \& Ultrasound, v. 44, $\mathrm{n}$. 1, p. 43-48, 2003.

WENCKE, M. W.; KIRBERGER, R. M. Radiographic gastrointestinal contrast study in the ostrich (Struthio camelus). Veterinary Radiology \& Ultrasound, v. 44, n. 5, p. 546-552, 2003.

WERTHER, K. Semiologia de Animais Silvestres. In: FEITOSA, F. L. (Ed.). Semiologia - a arte do diagnóstico. 2. ed.São Pulo: Roca, 2008, p. 655-718. 